This article was published as: Smart Materials and Structures 26 (9), 095028, 2017 DOI: https://doi.org/10.1088/1361-665X/aa7a87 


\title{
To the theory of elastic properties of isotropic magnetic gels.
}

\section{Effect of interparticle interaction.}

\author{
M.T.Lopez-Lopez ${ }^{\mathrm{a}, \mathrm{b}}$, L.Yu.Iskakova ${ }^{\mathrm{c}}$, A.Yu.Zubarev ${ }^{\mathrm{c}}$, B.Yu.Borin $^{\mathrm{d}}$ \\ ${ }^{a}$ Departamento de Física Aplicada, Facultad de Ciencias, Universidad de Granada, Campus de \\ Fuentenueva, 18071 Granada, Spain. \\ ${ }^{b}$ Instituto de Investigatacion Biosantaria ibs. GRANADA, Spain \\ ${ }^{c}$ Urals Federal University, Lenina Ave 51, 620083, Ekaterinburg, Russia \\ ${ }^{d}$ Chair of Magnetofluiddynamics, Measuring and Automation Technology, TU Dresden, 01069, Germany
}

\begin{abstract}
The paper deals with theoretical study of elastic shear properties of a magnetic gel, consisting of single magnetizable particles, homogeneously (gas-like) distributed in an elastic matrix. We suppose that the composite is placed in magnetic field, perpendicular to the direction of the sample shear. In order to get mathematically rigorous results, we have restricted ourselves by the analysis of the system with low concentration of the particles. Magnetic end elastic (through the matrix deformation) interaction between them is taken into account in the framework of the regular approximation of the pair interaction. Analysis shows that external magnetic field decreases macroscopic shear modulus of the composite with law concentration of the particles and increases this modulus if the particles concentration exceeds some threshold magnitude. The decreasing dependence of the modulus on the macroscopic shear is estimated. We believe that the suggested rigorous approach can be a robust background for the study of the systems with high concentration of the particles.
\end{abstract}

Key words: Magnetic gels; shear modulus; magnetic field effect

\section{Introduction.}

Magnetic gels are composites of fine magnetic particles in soft polymer matrixes. Coupling of rich set of physical properties of polymer and magnetic materials is very promising for many modern and perspective technologies. Discussions of technical and biomedical applications of these systems can be found, for example, in [1-12].

Macroscopic mechanic properties of these composites and effect of external magnetic field on these properties and behavior of these materials is actively discussed and studied problem. A short overview of works on this subject is given in [13].

Uniaxial elongation and magnetostriction effects in magnetic gels have been studied in many works (see, for example [13-19]). The shear deformations of these systems also present 
significant interest both from scientific and practical points of view. Theoretical studies of the shear effects in the composites with the particles, united in the linear chain-like aggregates, have been done in [20-22]. The general conclusion of these works is that an external magnetic field can significantly increase stiffnes of these composites.

The chain-like structures appear in magnetic polymers on the stage preceding the composite curing due to the action of an external magnetic field (field of polymerization). On the other hand, very often magnetic gels are prepared without the field. The spatial distribution of particles in these systems is rather random and isotropic (see, for example, $[15,17,23])$.

The aim of this work is theoretical study of effect of external magnetic field on the shear elastic modulus of magnetic gels with homogeneous and isotropic distribution of magnetic particles in the polymer matrix. It should be noted that many factors affect the macroscopic properties of the composite materials: shape of the particles; their magnetic properties and law of magnetization; the ratio of the particles size to the size of the host polymer cell; the chemical interaction between the particles with the polymer macromolecules. To achieve the deep insight of the microscopic nature of the macroscopic properties of magnetic polymers, these factors must be thoroughly studied.

In this work we focus on the effect of magnetic interaction between spherical, linearly magnetizable particles, randomly distributed in an elastic continuous media. This means, in part, we suppose that the size of the particles is much more than the size of the polymer cell. This situation is quite typical for the synthetic magnetic gels with micron-sized particles of the magnetic filler. We will suppose also that the macroscopic shear of the composite is small and the matrix obeys the linear law of deformations. Next, we assume that the gel is incompressible. It should be noted that the condition of the host polymer incompressibility is fulfilled not for all magnetic gels. However it allows us to restrict calculations and to get the final results in the transparent forms. Analysis of effects of the composite compressibility can be considered as a natural generalization of this model.

The principal and not overcome problem of the theory of composite materials is account of effects of multiparticle interactions [24]. Usually these interactions are taken into account by using various empirical and semi empirical approaches, whose accuracy, a' priory, is unknown.

In order to achieve mathematically rigorous results, we will consider the systems with moderate and low concentrations of the particles; effects of the interparticle interactions will be taken into account in the framework of the regular pair approximation. This approach allows us to avoid intuitive and heuristic theoretical constructions. That is why it can be 
considered as necessary robust background for the development of theoretical approaches for study of the concentrated systems where the multiparticle interactions are very significant.

\section{Physical model and main approximations.}

We consider a system of identical spherical non Brownian magnetizable particles embedded in an elastic continuous medium. For maximal simplification of the mathematics, we restrict ourselves by the analysis of the case of moderate or low magnetic fields and suppose the linear dependence of the particle magnetization on the local magnetic field.

Let us suppose that the composite is placed in a uniform magnetic field $\mathbf{H}$ and experiences the small shear deformation in the plane, perpendicular to the field (see Fig.1).

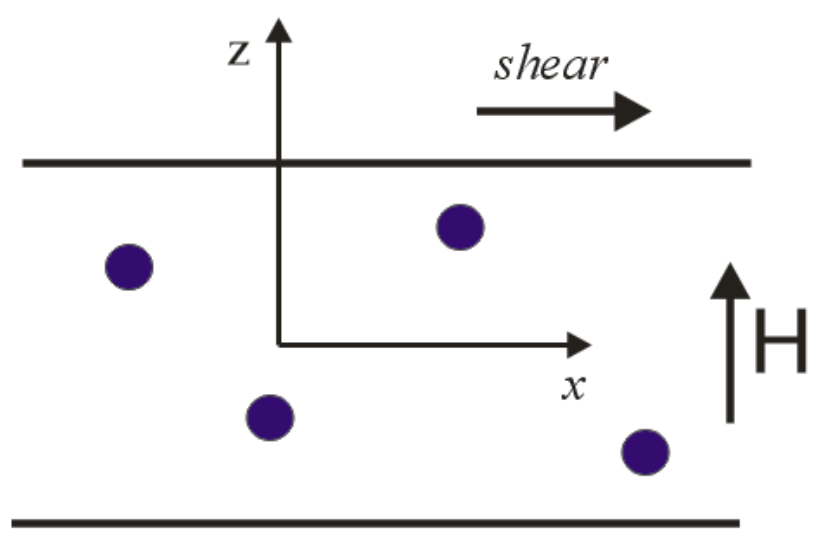

Fig.1. Illustration of the system under consideration.

It is convenient to introduce a Cartesian coordinate system with the axis $O z$ in the field direction and the axis $O x$ in the direction of the shear. By using the well known results of theory of magnetizable media (see, for example, $[25,26]$ ), as well as the similarity between the NavierStokes equation of a Newtonian incompressible fluid flow and the Lame equation of deformation of an elastic incompressible medium [24,], one can present the needed component of the macroscopic (measurable) stress $\boldsymbol{\sigma}$ in the composite as:

$\sigma=G_{e l} \gamma+\frac{1}{2} \varphi \mu_{0}<M_{x}>H$ 
Here $M_{x}$ is $x$ component of a particle magnetization, the angle brackets mean averaging over all relative positions of the particles, $G_{e l}$ is a part of the composite effective shear modulus, provided by the presence of the rigid spherical inclusions, $\gamma=\frac{\partial u_{x}}{\partial z}, u_{x}$ is the component of the macroscopic (measurable) vector $\boldsymbol{u}$ of the composite displacement, $\varphi$ is volume concentration of the particles, $\mu_{0}$ is the vacuum magnetic permeability. By using the Batchelor and Green results [27], for a low or moderately concentrated composites one can write down

$G_{e l}=G_{0}\left(1+2.5 \varphi+5.2 \varphi^{2}\right)$

where $G_{0}$ is the shear modulus of the pure polymer matrix. Usually this formula leads to good agreement with experiments till the concentrations $\varphi \sim 10-15 \%$.

One needs to note that, in principle, the solid particles can change the conformation of the macromolecules of the host polymer and, therefore, the matrix elastic modulus. The possibility of these transformations and their effect on the mechanic properties of the composites has been discussed, for example, in [28]. It was supposed that these transformations were induced by the chemical interaction of the ions, dissoluted from the particles surface, and the polymer macromolecules. Here we will neglect these effects and focus on the mechanic and magnetic interactions between the particles.

Our main goal now is to determine the component $\left\langle M_{x}\right\rangle$ of the particle magnetization. In order to get the mathematically rigorous results, we will estimate $\left\langle M_{x}\right\rangle$ taking into account interaction only between two particles, ignoring the effect of any third one. It should be noted that the Batchelor - Green formula (2) has been derived in the framework of the pair approximation, taking into account mechanic interaction between the particles through perturbations of the carrier medium.

Let us consider two particles and put the origin of the Cartesian coordinate system, shown in Fig.1, in the center of one of them. We will denote the radius vector of the center of the second particle by $\boldsymbol{r}$. This situation is illustrated in Fig.2; the coordinate axis $O y$ is not shown for simplicity. 


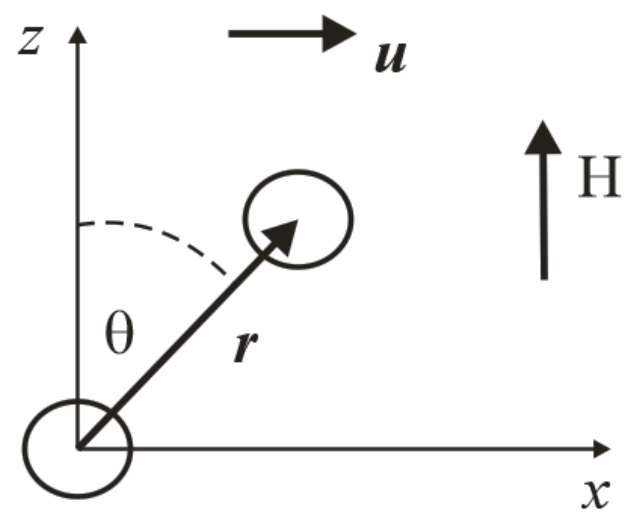

Fig.2. Two interacting particles and the used coordinate system.

The vector $\boldsymbol{u}$ of the macroscopic displacement of the composite is aligned along the axis $O x$, its gradient - in the $O z$ direction.

The component $M_{x}$ of magnetization of a particle, shown in Fig.2, appears because of magnetic interaction and mutual magnetization of the particles. The simplest way to take this interaction into account is to use the well known dipole-dipole approximation. However, this approximation describes quite well interaction between the particles only when they are far from each other and the distance $r$ between their centers significantly exceeds diameter $d$ of the particle. At the same time the effects of the mutual magnetization are especially strong at the particles close dispositions. At the small distances $r$ the interaction is multipolar. We will estimate the energy $U(\boldsymbol{r})$ of the particles magnetic interaction, by using the extrapolation formula, obtained in [29] from the results of numerical study of the problem on two linearly magnetizable particles:

$$
U=-3 \mu_{0} \mu_{f} H^{2} v \sum_{k=3}^{7}\left(\frac{\alpha-1}{\alpha+2}\right)^{p_{k}}\left[\frac{a_{k}}{\left(q-b_{k}\right)^{k}}+\frac{c_{k}}{\left(q-d_{k}\right)^{k}} \cos ^{2} \theta\right]
$$

Here $q=2 r / d ; \alpha=\mu_{p} / \mu_{f}$, where $\mu_{p}$ is magnetic permeability of the particle; $v=\pi d^{3} / 6$ is the particle volume; $p_{k}, a_{k} \ldots d_{k}$ are parameters, whose values are tabulated in [29]. For $q>>1$ the fit formula (4) coincides with the well known formula for the dipole-dipole approximation.

The $y$-component of the torque $\boldsymbol{\Gamma}$, acting on the cluster of the particles, can be calculated from two general relations. On the one hand $\Gamma_{\mathrm{y}}=-\frac{\partial U}{\partial \theta} \cos \phi[29,30]$; on the other one $\Gamma_{\mathrm{y}}=$ $-2 \mu_{0} v M_{x} H$ [26] (multiplier 2 appears here because we deal with a cluster, consisting of two particles). Equating these relations for $\Gamma_{\mathrm{y}}$, taking into account the relations $\mu_{f}=1, \alpha \gg 1$ are 
fulfilled for typical magnetic polymers, we find $M_{x}$ for the given relative disposition of the particles:

$M_{x}=3 H \sum_{k=3}^{7} \frac{c_{k}}{\left(q-d_{k}\right)^{k}} \sin \theta \cos \theta \cos \phi$

Here $\phi$ is the azimuth angle, not shown in Fig. 2 for brevity.

Let $g(\boldsymbol{r})$ be the function of statistic distribution over relative positions of the particles. For the convenience we suppose that the following normalization condition $g \rightarrow 1$ when $r \rightarrow \infty$ is held.

The in the frame of the pair approximation, the average $x$-component of a particle magnetization can be presented as:

$<M_{x}>=n \int M_{x}(\boldsymbol{r}) g(\boldsymbol{r}) d \boldsymbol{r}, n=\frac{\varphi}{v}$

Here $n$ is number of the particles in a unite volume of the composite. The distribution function $g$ can be presented as: $g=g_{0}+\delta g$, where $g_{0}$ corresponds to the non deformed composite; $\delta g$ reflects the change of the function because of the sample deformation. One can show easily that in the isotropic composite, where $g_{0}$ depends only on the absolute value $r$ of the radius vector $\boldsymbol{r}$, the equality

$\int M_{x}(\boldsymbol{r}) g_{0}(\boldsymbol{r}) d \boldsymbol{r}=0$

is held. Therefore:

$<M_{x}>=\frac{\varphi}{v} \int M_{x}(\boldsymbol{r}) \delta g(\boldsymbol{r}) d \boldsymbol{r}$

The function $\delta g$ can be determined from the following equation [31]:

$\delta g=-\operatorname{div}(g \boldsymbol{w})$

Here $\boldsymbol{w}$ is vector of the relative displacement of the particles. This vector can be determined by using the identity of the Navier - Stokes and Lame equations [24], as well as the relation [27] for the relative motion of two particles in a suspension. The result reads:

$$
\begin{aligned}
& w_{x}=\gamma z\left[1+(B-A) \frac{x^{2}}{r^{2}}-\frac{1}{2} B\right] \\
& w_{y}=\gamma \frac{x y z}{r^{2}}(B-A) \\
& w_{z}=\gamma x\left[(B-A) \frac{z^{2}}{r^{2}}-\frac{1}{2} B\right]
\end{aligned}
$$


Here $A(r)$ and $B(r)$ are functions of $r$, tabulated in [27]; $\gamma$ is the macroscopic dimensionless shear of the sample.

We suppose that $\gamma$ is small and present $\delta g$ in the form of the power series:

$\delta g \equiv g-g_{0}=\gamma q_{1}+\gamma^{2} q_{2}+\gamma^{3} q_{3}+\cdots$

Substituting (9) into (7), combining the terms with the same power of $\gamma$, one can get

$\gamma q_{1}=-\operatorname{div}\left(g_{0} \boldsymbol{w}\right)$

$\gamma^{2} q_{2}=-\gamma \operatorname{div}\left(q_{1} \boldsymbol{w}\right)=\operatorname{div}\left(\boldsymbol{w} \operatorname{div}\left(g_{0} \boldsymbol{w}\right)\right)$

$\gamma^{3} q_{3}=-\gamma \operatorname{div}\left(q_{2} \boldsymbol{w}\right)=-\operatorname{div}\left[\boldsymbol{w} \operatorname{div}\left(\boldsymbol{w} \operatorname{div}\left(g_{0} \boldsymbol{w}\right)\right)\right]$

By using (9) and (10) in (6), we come to the following relation:

$$
<M_{x}>=-\frac{\varphi}{v}\left\{\int M_{x}(\boldsymbol{r}) \operatorname{div}\left(g_{0} \boldsymbol{w}\right) d \boldsymbol{r}+\int M_{x}(\boldsymbol{r}) \operatorname{div}\left[\boldsymbol{w} \operatorname{div}\left(\boldsymbol{w} \operatorname{div}\left(g_{0} \boldsymbol{w}\right)\right)\right] d \boldsymbol{r}\right\}
$$

Taking into account that $M_{x} \sim r^{-3}$ and $\operatorname{div} w \rightarrow 0$ when $r \rightarrow \infty$, by using the OstrogradskiiGauss theorem, after some transformations one can present (11) in the form:

$$
\begin{aligned}
& <M_{x}>=-\frac{\varphi}{v}\left\{\int_{S} M_{x}(\boldsymbol{r}) g_{0} w_{r} d \boldsymbol{r}-\int_{V} g_{0}(w \cdot \nabla) M_{x}(\boldsymbol{r}) d \boldsymbol{r}+\int_{S} g_{0}(w \cdot \nabla)[(w\right. \\
& \left.\left.\nabla) M_{x}(r)\right] d \boldsymbol{r}-\int_{V} g_{0}(w \cdot \nabla)\left[(w \cdot \nabla)\left[(w \cdot \nabla) M_{x}(\boldsymbol{r})\right]\right] d \boldsymbol{r}\right\}
\end{aligned}
$$

This form is more convenient for calculations, than (11).

Here $S$ means the surface of a sphere with infinite radius and center in the center of the given particle; $V$ is volume of this sphere.

\section{Results and discussion.}

It is convenient to use the spherical coordinate system $(r, \theta, \phi)$ with the origin in the center of one of the particles, shown in Fig. 1 and the polar axis aligned along $O z$. The following relations between the Cartesian and the spherical components of the vector $\boldsymbol{r}$ are true:

$x=r \sin \theta \cos \phi, \quad y=r \sin \theta \sin \phi, \quad z=r \cos \theta$

In this coordinate system, the components of the vector $\boldsymbol{w}$ read: 
$w_{r}=\gamma r(1-A) \sin \theta \cos \theta \cos \phi$

$w_{\theta}=\gamma r\left(\cos ^{2} \theta+\frac{1}{2} B\left(\sin ^{2} \theta-\cos ^{2} \theta\right)\right) \cos \phi$

$w_{\phi}=-\gamma r\left(1-\frac{1}{2} B\right) \cos \theta \sin \phi$

Note that the equality $A(r)=1$ is held at $r=d$ [27]. Thus the condition $w_{r}(r=d)=0$ of the particles non intersection is fulfilled in (13). At the same time the components $w_{\theta}$ and $w_{\phi}$ are not zero at $r=d$. This means that the particles can slip over each other being in the physical contact.

We will chose the distribution function $g_{0}$ of the hard spheres in the non deformed composite by using the simplest form which takes into account the steric interaction between the particles and the short range order, created by this interaction [32] :

$$
g_{0}=1+8 \varphi\left(\begin{array}{c}
0, r<d \\
\left.1-\frac{3 r}{4 d}+\frac{r^{3}}{16 d^{3}}\right), d<r<2 d \\
1, r>2 d
\end{array}\right.
$$

Combining (12,13,) and (14), after simple but cumbersome calculations we get:

$\frac{1}{2} \varphi \mu_{0}<M_{x}>H=\mu_{0} \varphi^{2} H^{2}\left(Q_{1}-Q_{2} \gamma^{2}\right) \gamma$

$Q_{1} \approx(-0.417+0.614 \varphi) ; \quad Q_{2} \approx 9.6-1.26 \varphi$

Substituting (15) into (1), one can rewrite the last relation as:

$\sigma=G \gamma$

$G=G_{e l}+G_{m}$

Here $G$ is the elastic shear modulus of the composite, the modulus $G_{e l}$ is estimated in eq. (2) and

$$
G_{m}=\mu_{0} \varphi^{2} H^{2}\left(Q_{1}-Q_{2} \gamma^{2}\right)
$$

Parameter $G_{m}$ reflects the contribution of the magnetic effects to the shear modulus $G$. Since the derivation of (17) is based on the power expansion (9), this relation is fulfilled only for small deformations, when the inequality $Q_{1}>Q_{2} \gamma^{2}$ is held.

The relations $(15,16)$ show that $G_{m}$ is negative for small concentrations $\varphi$ and can be positive only when $\varphi$ is large enough. Therefore, the total shear modulus $G$ must decrease with magnetic 
field in the low concentrated composites and increase with the field when the concentration exceeds some critical magnitude. The microscopic physical cause of this, might, unexpected result, is illustrated in Fig. 3.

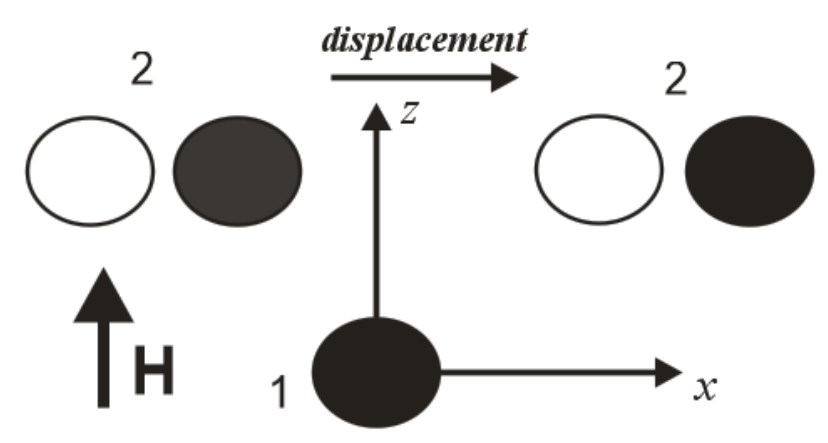

Fig. 3. Sketch of the interacting particles. White and black circles - positions of the particle 2 before and after the macroscopic shear respectively.

Let us suppose that we determine the component $M_{x}$ of the particle 1 in Figure 3 . This component appears due to magnetic interaction between the particle 1 and some particle 2 . Because the initial spatial distribution of the particles is isotropic, the particle 2, with the equal probabilities, can be situated either left, or symmetrically right from the axis $O z$. Obviously, if the particle 2 is left from this axis, the magnetization vector $\mathbf{M}$ of the first particle deviates left (i.e. $M_{x}$ is negative); if the particle 2 is situated right from $O z$, the component $M_{x}$ is positive. Bcause of the symmetrical positions of the second particles in the non deformed sample, the resulted value of $M_{x}$, before deformation, is zero. However after the shear, the left particle 2 becomes closer to the particle 1 , than the right particle. That is why its influence on the magnetization $\mathbf{M}$ is stronger than effect of the right particle. As a consequence, the resulted vector $\mathbf{M}$ is deviated left from the axis $O z$, i.e. the resulted component $M_{x}$ is negative. This leads to the negative sign of the first term in the relation (15) for $Q_{1}$.

The second term $614 \varphi$ in this relation takes place due to the term, proportional to $\varphi$, in the formula (14) for $g_{0}$. This term reflects the interparticle correlations in non ideal gas of hard spheres [32]. Because of these correlations, the distribution function (14) has a maximum at $r=d$ (i.e. when the particles are in the direct contact). In this position the particles can slip over each other $\left(w_{r}=0, w_{\theta}, w_{\phi} \neq 0\right.$ at $\left.=d\right)$. Analysis shows that this slip leads to the resulted inclination right of the magnetization $\mathbf{M}$, therefore, to the positive sign of the resulted component $M_{x}$. That is why, the term, proportional to $\varphi$ in the relation for $Q_{1}$, is positive. Since the maximum of the distribution function $g_{0}$ increases with $\varphi$, the effect of the particles slip enhances and second term in the relation for $Q_{1}$ dominates when $\varphi$ is high enough. 
It should be noted that the approximation (4) corresponds to the gas of hard spheres with moderate or low concentrations of the particles. For higher concentrations more complicated relations for $g_{0}$ must be used. Unfortunately, their explicit analytical forms are not determined; as a rule, these functions are calculated from solution of special non-linear integro-differential equations (see discussion in [32]).

The relations $(15,16)$ demonstrate that the term $G_{m}$, therefore, the total shear modulus $G$ decreases with the macroscopic shear $\gamma$, even when the elastic matrix obeys the linear law of deformation. Decreasing dependences of the composite shear modulus on the shear strain have been detected in experiments $[33,34]$.

\section{Discussion.}

We present results of analysis of effect of magnetic and elastic (through the host matrix) interactions between spherical magnetizable particles, randomly distributed in the matrix, on the macroscopic shear modulus of magnetopolymer composites. Our analysis is based on the mathematically rigorous two-particle approach and does not contain any intuitive or heuristic construction. The results show that, in the low concentrated composites, the interparticle interaction leads to decreasing dependence of the shear modulus on the applied magnetic field. In the systems with high enough concentration of the particles, one can expect increase of the modulus with the field. The effective shear modulus decreases with the macroscopic shear of the composite.

The accurate estimate of the threshold concentration, corresponding to the inversion of the dependence of the modulus on the field, requires solution of multiparticle problem. That is rather impossible by using only analytical methods of theoretical physics, however can be done by using the modern methods of computer simulations.

One needs to note that the conclusion on the decreasing dependence of the modulus on the applied field is is a direct consequence of the assumptions on the random, gas-like spatial distribution of the magnetically soft spherical particles. In the real magnetic gels these effects can be masked by the non-spherical shape, at least, of a part of the particles; by the presence of magnetically hard particles with some remnant magnetization; by anisotropic agglomerates (say, doublets), consisting of the particles, linked by non magnetic forces. It should be noted that these agglomerates very often appear on the stage of the gels synthesis. All these factors must lead to the mechanical rotation (turn round) of the particles (agglomerates) under the action of the 
macroscopic shear. Preliminary estimates show that this rotation effect must lead to increase of the composite shear modulus with the applied field.

We believe that the deep insight in the problem of determination of macroscopic properties of the magnetic composites requires detailed analysis of various microscopic factors which affect these properties. Here we present the results of study of one of them (effects on the interparticle interaction). Analysis of the other ones can be subject of separate works.

\section{Acknowledgements.}

L.Iskakova and A.Zubarev are grateful for the financial support to the Russian Fund for Basic Research, 16-58-12003; the Program of Russian Federation Ministry of Science and Education, project 3.1438.2017/PCh.

M.L-L was supported by the project FIS2013-41821-R (Plan Nacional de Investigación Científica, Desarrollo e Innovación Tecnológica, Ministerio de Economía y Competitividad, Spain, co-funded by ERDF, European Union).

D. Borin would like to acknowledge the support of DFG (Deutsche Forschungsgemeinschaft) under Grant Bo 3343/1-1.

\section{References}

1. Filipcsei G., Csetneki I., Szilagyi A.and Zrınyi M., 2007 Adv. Polym. Sci., 206, 137

2. Boczkowska A.and Awietjan S.F., Materials Science Forum , 2010, 636-637, 766

3. van Bruggen M. and van Zon J.,2010, Sens. Actuators A Phys., 158, 240

4. van Berkum S., Dee J.T., Philipse A. P. and Erné B.H., 2013 Int. J. Mol. Sci., 14, 10162

5. Bañobre-López M., Piñeiro-Redondo Y., de Santis R., Gloria A., Ambrosio L., Tampieri A., et al. 2011 J Appl Phys., 109, 07B313

6. Das B., Mandal M., Upadhyay A., Chattopadhyay P., Karak N., 2013 Biomed. Mater., 8, 035003

7. Gloria A., Russo R., d'Amora U., Zeppetelli S., d'Alessandro T., Sandri M., et al., 2013 J. R. Soc Interface, 10, 20120833

8. Li Y., Huang G., Zhang X., Li B., Chen Y., Lu T., et al, 2013 Adv Funct Mater. 23, 660

9. Panseri S., Cunha C., Alessandro T., Sandri M., Giavaresi G., Marcacci M., et al., 2012, J Nanobiotechnology, 10, 32 
10. Zeng X.B., Hu H., Xie L.Q., Lan F., Jiang W., Wu Y., et al., 2012, Int J Nanomed., 7, 3365

11. Singh R.K., Patel K.D., Lee J.H., Lee E.J., Kim J.H., Kim T.H., et al., 2014, PLOS ONE, 9, e91584

12. Lopez-Lopez M. T., Scionti G., Oliveira A.C., Duran J.D. G., Campos A., Alaminos M., Rodriges I.A., 2015, PLOS ONE, DOI:10.1371/journal.pone.0133878 July 24,

13. Lopez-Lopez M. T., Durán Juan D. G., Iskakova L. Yu. and A. Yu. Zubarev, 2016, Journal of Nanofluids, 5, 1-17,

14. Kchit N., Lancon P.and Bossis G., 2009, J.Phys.D.Appl.Phys. 42, 105506

15. Stepanov G. V., Abramchuk S. S., Grishin D. A., Nikitin L. V., Kramarenko E. Y. and A. Khokhlov R., 2007 Polymer, 48, 488

16. Shen Y., Golnaraghi M. F. and Heppler G. R., 2004 , J.Intell. Mater. Struct, ,15,27

17. Stepanov G., Borin D., Raikher Yu., Melenev P.and Perov N.S., 2008, J. Phys.: Condens. Matter, 20204121

18. Diguet G., Beaugnon E.and Cavaille J. Y., 2010 J. Magnetism and Magnetic Materials 322,3337

19. Romeis D., Toshchevikov V. and Saphiannikova M., 2016, SoftMatter, 12, 9364

20. Jolly M.R., Carlson J. D., Muñoz B. C., Bullions T.A., 1996, Journal of Intelligent Material Systems and Structures, 6, 613-622

21. Danas K., Kankanala S.V.and Triantafyllidis N., 2012, J. Mechanics and Physics of Solids, 60120

22. Han Y., Hong W., Faidley L.E., 2013, International Journal of Solids and Structures, 50 $2281-2288$

23. Khimi S. R., Pickering K.L., 2015, Compos. Part B Eng. 83, 175

24. Cristensen R., 1979, Mechanics of Composite Materials, New York: Wiley.

25. Rosensweig R., Ferrohydrodynamics. 1985, Cambridge University Press, Cambridge, New York,

26. Landau L.D., Lifshitz E.M., 1960, Electrodynamics of Continuum Media, Pergamon Press, London,

27. Batchelor G.K., Green J.T., 1972, J.Fluid Mechanics, 56 (3), 4101

28. Borin D., Günther D., Hintze C., Heinrich G. , Odenbach S., J. Magnetism and Magnetic Materials, 2012, 324 3452-3454

29. Biller A. M., Stolbov O. V. and Raikher Yu. L., 2014, J. Appl. Phys., 116, 114904.

30. Landau L., Lifshitz E., 1970, Theory of Elasticity, Pergamon Press, London. 
31. Shkel Y., Klingenberg D., 1998 J.Appl.Phys., 83, 7834

32. Balesku R., 1975 Equilibrium and non equilibrium statistical mechanics, V.1. WileyInterscience publication. John Wiley and Sons.

33. Shen Y, Golnaraghi M F, Heller G R, , 2004, J. Intelligent Materials Systems and Structures. 15, 27

34. Schubert G, Harrison P, 2015, Polymer Testing 42, $122 \mathrm{e} 134$. 